\title{
Desarrollo de la atención selectiva a través del juego en estudiantes de educación superior
}

\section{Development of selective attention through play in higher education students}

\author{
Blanca Carpio Lozada \\ Universidad San Martín de Porres-Filial Arequipa, Arequipa, Perú. \\ bcarpiolozada@hotmail.com
}

Recibido: 23/05/2020

Aceptado: 25/10/2020

\section{Resumen}

La investigación está enfocada en la aplicación de los Juegos "DIMAPA" que son un conjunto de actividades dinámicas, entretenidas, motivadoras y pautadas, basadas en dibujos, mandálas y palabras, diseñadas para el curso de Histología y Embriología General y Aplicada. El objetivo del artículo es desarrollar la atención selectiva de los estudiantes de Odontología. El método de investigación es de tipo aplicada, con un nivel explicativo, con diseño cuasi-experimental. La muestra del grupo experimental esta constituida por 13 estudiantes y el de control por 11, sobre los cuales se aplicó el test de atención d2; luego, al grupo experimental se le aplicaron los juegos "DIMAPA" en 30 sesiones de aprendizaje, distribuidas en 4 por semana. Culminada la intervención se procedió a aplicar nuevamente el test de atención d2 a los dos grupos conformados y se procedió al procesamiento de los datos obtenidos. En el análisis e interpretación de los resultados se demuestra que, según la prueba estadística $\mathrm{t}$ de Student, los estudiantes a quienes se les aplicó los juegos "DIMAPA", aumentaron significativamente el desarrollo de su atención selectiva, de acuerdo a los tres parámetros evaluados (efectividad total: $\mathrm{p}=0.034$, concentración: $p=0.023$ y variación: $p=0.048$ ). Se concluye que los juegos "DIMAPA" desarrollan la atención selectiva.

Palabras clave: Juegos "DIMAPA", Atención Selectiva y Aprendizaje.

\begin{abstract}
The research is focused on the application of the "DIMAPA" Games, which are a set of dynamic, entertaining, motivating and guided activities, based on drawings, mandalas and words designed for the General and Applied Histology and Embryology course; being the main objective of the work to develop the selective attention of the students of Dentistry. This is an applied type investigation, with an explanatory level, an experimental study with a quasi-experimental design and a quantitative approach. The sample of the experimental group consisted of 13 students and the control by 11 , on which the attention test d2 was applied; after which the "DIMAPA" games were applied to the experimental group in 30 learning sessions, distributed over 4 per week. After the intervention, the $\mathrm{d} 2$ attention test was applied again to our two formed groups and the data obtained was processed. In the analysis and interpretation of the results, it was shown that, according to the Student's t test, the students to whom the "DIMAPA", games were applied significantly increased the development of their selective attention, according to the three parameters evaluated (effectiveness total: $\mathrm{p}=$ 0.034 , concentration: $p=0.023$ and variation: $p$ $=0.048$ ), therefore we conclude that the games "DIMAPA" develop selective attention; For this reason, the proposed alternative hypothesis is accepted rejecting the null $(\mathrm{p}<0.05)$.
\end{abstract}

Keywords: "DIMAPA" Games, Selective Attention and Learning. 


\section{Introducción}

La atención selectiva, es básicamente una función mental que procesa parte de una información, es decir, discrimina lo interesante del estímulo; ello indica que el proceso de selección de estímulos está unido a la atención, de tal manera que cuando un estímulo es seleccionado por algún motivo, este será el que pase el filtro de la atención nuestra.

En el estudio realizado durante las actividades teórico-prácticas desarrolladas en el aula; se logró evidenciar que uno de los problemas que afecta a los estudiantes universitarios, está relacionado con dificultades de atención selectiva; pues tienden a distraerse con facilidad, no permitiéndoles concentrarse en tareas específicas, generando así dificultades en la asimilación de conocimientos y por tanto en un aprendizaje significativo.

La atención selectiva cobra gran importancia a nivel académico (Stevens y Bavelier, 2012, p. 30). Cual sea el nivel educativo, la atención juega un papel determinante en el rendimiento académico; es así que los jóvenes universitarios con problemas de atención, pueden tener un rendimiento académico muy bajo -entre otras consecuencias (Barragán, et al. 2007, p.23). Conduciendo al estudiante al fracaso académico que se ha convertido en una seria preocupación para las instituciones de educación superior (Khan, et al. 2013, p.80). Es así que el desafío común en el campo educativo es reducir este fracaso (Cid-Sillero, et al. 2020, p.60).

De los argumentos definidos; la atención selectiva es una herramienta para poder interpretar y seleccionar la información disponible, y es en la actividad educativa en nuestro caso que pone en marcha y controla todos los procesos y mecanismos por los cuales el organismo procesa tan solo una parte de toda la información, y da respuesta solo a aquellas demandas del ambiente que son útiles o de gran importancia para el estudiante.

Dentro del ámbito de la neuroeducación, las funciones ejecutivas han cobrado especial interés en los últimos años, por haberse demostrado su relación con el rendimiento académico (Véglia y González, 2018, p.28). Estas funciones coordinan la actividad de las funciones mentales superiores, tales como la atención y dentro de sus modalidades encontramos a la atención selectiva, la cual exige un mayor esfuerzo cognitivo. En efecto, las funciones ejecutivas y la atención son imprescindibles para realizar con éxito cualquier aprendizaje (Portellano, 2019, p.112).

De ello se infiere que la atención selectiva, es la capacidad de focalizarlamente en unestímulo o tarea concreta, a pesar de otros estímulos ambientales, y es ahí donde el estudiante universitario debería dar preferencia a determinados estímulos capaz de atender los más relevantes e inhibir los distractores.

Siendo además una de las grandes preocupaciones de la educación superior, determinar en qué punto del procesamiento cognitivo se produce la acción del proceso selectivo en el estudiante; ya que nuestro subconsciente trabaja continuamente, sin que se le note, descartando de manera automática todos aquellos estímulos que considera poco importantes.

En relación a lo expuesto cabe resaltar la importancia de la neurociencia en el contexto educativo, ya que permiten conocer de manera científica nuestra actividad cerebral (Cherrier, et al. 2020). Y como parte de las neurociencias, la neuroeducación, se ocupa específicamente de estudiar la optimización del proceso de enseñanza - aprendizaje con base en el funcionamiento del cerebro y los fundamentos neurobiológicos que lo sustentan (Gago-Galvagno y Elgier, 2018, p. 40). De tal manera que, la neuroeducación contribuye a la innovación y transformación de la educación y su práctica pedagógica; en ayuda de la neurodidáctica y sus estrategias metodológicas para facilitar el aprendizaje de los educandos en pos de lograr una educación para la vida (Falconi, et al. 2017, p. 61).

Este es el motivo del estudio de promover el juego como estrategia para ver el proceso de la atención selectiva de manera directa en los estudiantes universitarios; dada la importancia de las funciones ejecutivas en el aprendizaje y por ende en el rendimiento académico, es necesario implementar estrategias educativas que ayuden a estimular dichas funciones. (Véglia y González, 2018, p. 30).

Por tanto, el juego y/o las técnicas lúdicas en la presente investigación se convierten en mediadores para desarrollar la atención selectiva que mejoraría el aprendizaje en los estudiantes universitarios, a través de experiencias agradables de tal manera logren competencias y capacidades para la comprensión de conceptos teóricos en el ámbito académico. 
Por tal motivo, surge la inquietud de implementar el juego como estrategia, como son los juegos "DIMAPA" para desarrollar la atención selectiva que es una función mental superior; estos juegos al mismo tiempo actúan como mediadores en los procesos de enseñanza aprendizaje logrando en los estudiantes universitarios una mejor comprensión de conceptos, categorías y teorías, optimizando así, su rendimiento académico.

El estudio tiene como objetivo determinar la influencia del juego en el desarrollo de la atención selectiva de los estudiantes de odontología de una Universidad Privada de Arequipa, para mejorar el aprendizaje y asimilación de conocimientos teóricos.

\section{Marco teórico}

\section{La atención selectiva}

Según, Introzzi et al. (2019), definen la atención selectiva como: "una función cognitiva que orienta la atención hacia un objeto o estímulo que resulta relevante, evitando la distracción ante aquellos que son irrelevantes" (p. 106).

Es decir, laatención selectiva, permitea unindividuo seleccionar y enfocarse en algo en particular para su posterior procesamiento, mientras que al mismo tiempo suprime información irrelevante o que lo distrae, esta capacidad de concentrarse en la tarea en cuestión y de ignorar la distracción, parece tener efectos relevantes en varios dominios importantes a fundamentos académicos, (Stevens y Bavelier, 2012, p. 33).

Entonces la atención selectiva ayuda a mejorar el rendimiento cognitivo, mejora las experiencias de aprendizaje y filtra la información útil: "La atención selectiva y las funciones ejecutivas, son un conjunto de habilidades mentales que permiten dar solución a nuevas situaciones y problemas frente a los que no disponemos de conocimiento previo" (Portellano, 2019, p.110).

Estas funciones coordinan la actividad de las funciones mentales superiores tales como la atención. En efecto, las funciones ejecutivas y la atención forman un binomio inseparable e imprescindible para realizar con éxito cualquier aprendizaje y sin su correcto funcionamiento sería muy difícil conseguir una adecuada conducta adaptativa.
Siguiendo con el tema, Portellano (2019), distingue dos modalidades de atención según el grado de complejidad: involuntaria y voluntaria o ejecutiva; esta última se refiere de manera muy especial a las modalidades atencionales que exigen un mayor esfuerzo cognitivo, dentro de ellas se encuentra la atención selectiva; definiéndola como la capacidad para mantener una determinada respuesta ante un estímulo a pesar de la presencia de otros estímulos distractores que de manera simultánea compiten entre sí; permitiendo prestar atención a estímulos relevantes, excluyendo otros.

La atención selectiva por tanto es la actividad que ejecuta y controla todos los procesos por los cuales la mente selecciona la información; donde el tiempo de concentración depende del interés que genere la tarea y de la dificultad de la misma.

Cid, et al., (2020) consideran que, entre las funciones ejecutivas, la atención merece una consideración especial, ya que representa el pilar fundamental sobre el que descansa todo el aprendizaje. Por este motivo la atención del alumnado parece tener una influencia directa sobre su rendimiento académico, es decir, aquellos que presentan un mejor desempeño ejecutivo suelen obtener mejores calificaciones (Jiménez, et al. 2019, p. 215). Rojas y Rincón (2015). es también, la capacidad para seleccionar, de entre varias posibles, la información más relevante ya que la atención selectiva procesa solo parte de una información (p.44)

Se infiere que la atención selectiva es de uso más frecuente, pero no todas las personas logran la concentración adecuada; ya que es una habilidad que se aprende y alcanza en la medida que se implementen estrategias para el uso eficiente de las funciones mentales superiores $\mathrm{y}$ procesos cognitivos.

\section{El juego como técnica lúdica}

A lo largo de la historia, el juego y el aprendizaje han estado íntimamente ligados; son numerosas las teorías y escuelas que parten del juego como base ideal del aprendizaje. Desde la antigüedad hasta la actualidad el juego está presente a lo largo de toda la vida del hombre (Calvo y Gómez, 2018, p. 25).

Por su parte Huizinga (1972) uno de los teóricos más importantes del juego, considera que necesitamos permanentemente del juego para la 
vida, ya que nos brinda alegría y motivación; al mismo tiempo la vivencia del juego proporciona un enriquecimiento integral del ser humano.

Para este autor lo lúdico está en la naturaleza humana, forma una parte profunda y espontanea de la vida emocional del hombre. Bajo este enfoque entonces, se tiene como fin el desarrollo de las funciones mentales superiores como la atención a través del juego.

De la misma manera De la Cruz, et al. (2020), resaltan el poder de los juegos y que jugar puede ser tan serio como vivir, aunque se viva como un juego; jugar revela el carácter de los jugadores y lo que traen en mente. Siguiendo la misma postura, Vergara (2017) considera que el juego es como un ejercicio para la vida y un agente natural educativo

Es una herramienta que forma parte no sólo en la vida de los niños, sino que también del adulto, el juego forma parte del diario vivir, siendo visto como una manera para recrearse, divertirse, desestresarse, aprender, entre otras (Montero, 2017, p. 77). Favorecen el mantenimiento del estado cognitivo y funcional de las personas mayores, facilitando el aprendizaje significativo y la interacción social (Souza, et al. 2016, p.155).

Asimismo, Girgin (2017) considera que, no importa la edad para jugar y que los que pasan su tiempo jugando están por delante de los que no lo hacen; además, propone que se deben de crear laboratorios de juegos y juguetes en escuelas y universidades, abrir cursos de desarrollo de juegos en las facultades de educación y programas de posgrado para que los aspirantes a docentes puedan tomar cursos para desarrollar juegos. Es así que el juego es considerado fundamental en el desarrollo de las personas como construcción individual y social, estimulando el desarrollo físico-motor, permitiendo la interacción social, estimulando valores, actitudes y normas necesarias para la armonía social (Mendieta, et al. 2019, p.15).

Los beneficios del aprendizaje basado en juegos están bien justificados en la literatura, pero no existen suficientes estudios sobre sus aplicaciones en diferentes disciplinas en la Educación Superior; sin embargo, los resultados empíricos apoyan la eficacia del juego en la educación superior y también muestran que los estudiantes disfrutan con este tipo de aprendizaje. (Mas-Machuca, et al. 2019, p. 57)
Siendo el juego una herramienta esencial en la práctica docente para promover el aprendizaje; la implementación de estrategias lúdicas en el contexto universitario constituye un método innovador y ameno de enseñanza al proporcionar mejores experiencias de aprendizaje, debido a que despierta el interés de los estudiantes y los invita a reflexionar y utilizar la creatividad en la formulación de posibles soluciones a problemas (Marles, et al. 2018, p.50).

De la misma manera para Flores (2016); la importancia del desarrollo de la atención selectiva a través del juego está ligada al desafío y actividad intensa del aprendizaje para poder generar mejores resultados en la adquisición de conocimientos en el ámbito de la educación (p. 190). Según describe sobre los juegos Gargallo (2000) es que; teniendo en cuenta que, la estimulación cognitiva optimiza la atención, es que se centra la presente investigación a través, de la aplicación de juegos; inspirados en el programa de intervención educativa. Estos juegos "DIMAPA" son un conjunto de actividades dinámicas, entretenidas, motivadoras y pautadas, basadas en dibujos, mandálas y palabras, diseñadas con relación al curso de histología y embriología de la carrera de Odontología.

\section{Metodología}

Elmétodo de investigación es científico. Para Bunge (1976) "El método científico es un procedimiento para tratar un conjunto de problemas, donde cada uno de los problemas requiere un conjunto de métodos y técnicas especiales" (p.24). por lo que se formula el problema con precisión sobre la realidad observada y la teoría existente, proponiendo suposiciones bien definidas y fundadas, y se somete la hipótesis a contrastación dura, no laxa, e indaga porque el resultado es como es. El nivel de investigación es aplicado, para Arias (2012) busca contestar porqué sucede determinados fenómenos, cual es la causa; las preguntas centrales de este nivel de estudio son: que y porque ocurren estos hechos o fenómenos; para ello desarrolla dos características de estudio: la observación y el experimental. Se utilizó el tipo de investigación aplicada, y según Baena (2017) busca conocer una realidad concreta de los problemas sociales, para modificar o generar conocimiento, ocupándose del proceso de relación entre la teoría y los resultados. La investigación corresponde al enfoque cuantitativo que es un conjunto de procesos secuenciales y probatorios; y es de tipo experimental con diseño 
cuasiexperimental, para Hernández et al. (2014) es que, en el diseño cuasiexperimental los sujetos no se asignan al azar a los grupos ni se emparejan, sino que, ya están formados antes del experimento; son dos grupos con características semejantes, en el grupo experimental se realiza la intervención, en el de control, no. Sin embargo, a ambos grupos se aplica la pre y posprueba.

La población censal, se constituyó por la totalidad de estudiantes del segundo semestre de Odontología, matriculados en el curso de Histología y Embriología General y Aplicada, de esta se obtuvo una muestra no probabilística constituido por 10 mujeres y 3 varones, haciendo un total de 13 estudiantes para el grupo experimental $\mathrm{y}$, por 11 personas, todas mujeres para el grupo control a quienes se le aplicó el pre-tets y post-tets; las edades de los participantes de ambos grupos oscilan entre 17 a 18 años, por lo que podemos afirmar que la edad y sexo en ambos grupos se distribuyen homogéneamente, no influyendo estas características en los resultados finales. La técnica utilizada fue la observación directa. El estudio fue realizado en su totalidad en el laboratorio de prácticas. Al inicio del semestre se procedió a la aplicación del instrumento de medición del estudio en un tiempo aproximado de 10 minutos, utilizando el test de atención d2. Esta es una prueba de cancelación de letras, que determina la capacidad de prestar atención a un estímulo o hecho, mientras suprime las letras distractoras (Gall et al. 2018, p. 17). Por lo tanto, mide la atención selectiva asociada con la atención sostenida y se aplica en edades de 8 años en adelante (Cedeño y Loor, 2019, p.35).

Se aplicó los juegos "DIMAPA" en 30 sesiones de aprendizaje, distribuidas en 4 por semana. En cada una de ellas se brindó al estudiante las indicaciones, normas y/o reglas de juego respectivas, que contribuyan con el entendimiento y desarrollo adecuado de cada sesión de juego; luego se procedió con el desarrollo de la actividad en el tiempo determinado según cada sesión, después se realizó la evaluación de las actividades, las que fueron adjuntadas en una ficha de seguimiento. Culminadas las sesiones, se volvió a aplicar el test de atención $\mathrm{d} 2$ y se procedió al procesamiento de los datos obtenidos, utilizando la versión clásica del test (lápiz y papel). Con los datos numéricos recabados, obtenemos las tres dimensiones del test d 2 correspondiente a nuestra variable dependiente; nos referimos a:
- Efectividad total (TOT). Es la medida principal para la validación del test $\mathrm{d} 2$ y la que más se utiliza en estudios experimentales y aplicados; proporcionando una medida de control atencional e inhibitorio y de relación entre la velocidad y precisión de los sujetos.

- Índice de concentración (CON). Esta puntuación es muy fiable y proporciona un índice del equilibrio entre la velocidad y la precisión en la actuación de los sujetos.

- Indice de variación o diferencia (VAR). La puntuación VAR evalúa la estabilidad y la consistencia en el tiempo de la actuación del sujeto. Por ello es importante resaltar que una puntuación VAR extremadamente alta puede sugerir una inconsistencia en el trabajo del sujeto.

Siguiendo la teoría de Brickenkamp (2009). Las puntuaciones efectividad total (TOT) e índice de concentración (CON) se interpretan de modo directo, es decir; cuanto mayor es la puntuación, mayor es la capacidad de atención del estudiante; por el contrario, las puntuaciones de índice de (VAR) conforme más baja sea esta puntuación es mejor dado que una puntuación VAR extremadamente alta puede sugerir una inconsistencia en el trabajo del sujeto. Sabiendo que los valores mínimos de las tres dimensiones es 0 y valor máximo para efectividad total (TOT) es 658, concentración (CON) es 299 y variación (VAR) es 47.

Para el análisis estadístico se empleó la prueba paramétricat de Student, cuya finalidad es comparar dos medias aritméticas, siendo en nuestro estudio el pre-test y post-test, entre los grupos de estudio.

Esta investigación se desarrolló en el año 2018; con el objeto de estudio, para mejorar la atención selectiva a través de los juegos "DIMAPA" en los estudiantes de odontología de una Universidad Privada en la ciudad de Arequipa.

\section{Resultados}

Se comprobó la eficacia de los juegos "DIMAPA" al comparar las puntuaciones de los estudiantes tanto del grupo control como del grupo experimental en dos momentos: antes y después de la administración del programa aplicado al grupo experimental. Y para el análisis estadístico se empleó la prueba del T de Student, considerando significancia estadística a los valores menores a 
0.05 , tanto para el pre-test y post-test como para la contrastación entre los dos grupos de estudio (de control y experimental). Los resultados obtenidos fueron los siguientes:

Tabla 1.

Comparación de la Atención Selectiva pre-test entre el Grupo control y Experimental

\begin{tabular}{llll}
\hline \multirow{2}{*}{$\begin{array}{c}\text { Atención Selectiva } \\
\text { Medición Pre-test }\end{array}$} & \multicolumn{2}{c}{ Grupo de Estudio } \\
\cline { 2 - 4 } Efectividad Total & Media Aritmética & 413.91 & 470.15 \\
& Valor Mínimo & 306 & 314 \\
& Valor Máximo & 593 & 569 \\
\cline { 2 - 4 } & $\mathrm{P}$ & $0.117(\mathrm{P} \geq 0.05)$ N.S. \\
\hline \multirow{3}{*}{ Concentración } & Media Aritmética & 154.00 & 173.00 \\
& Valor Mínimo & 88 & 71 \\
& Valor Máximo & 259 & 240 \\
\cline { 2 - 4 } Variación & P & $0.338(\mathrm{P} \geq 0.05)$ N.S. \\
\hline \multirow{3}{*}{ Media Aritmética } & 19.64 & 18.62 \\
& Valor Mínimo & 9 & 8 \\
& Valor Máximo & 43 & 33 \\
\cline { 2 - 4 } & P & $0.769(\mathrm{P} \geq 0.05)$ N.S. \\
\hline
\end{tabular}

Matriz de datos recolectados.

En la tabla 1 con respecto a la atención selectiva pre-test entre el grupo control y grupo experimental se puedo observar que, en cuanto a la efectividad total, los estudiantes del grupo control obtuvieron una puntuación de 413.91; mientras que los estudiantes del grupo experimental obtuvieron una puntuación de 470.15; ésta diferencia estadísticamente no es significativa. Lo mismo sucede con la concentración, los estudiantes del grupo control obtuvieron un puntaje de 154 y los del grupo experimental 173, de ello se ${ }_{36}$ infiere que, la diferencia de puntuaciones, no es significativa estadísticamente. Con respecto a la variación, sabiendo que conforme más baja sea esta puntuación es mejor; los estudiantes del grupo control obtuvieron un puntaje de 19.64 y los del grupo experimental 18.62, a pesar de que los estudiantes del grupo experimental obtuvieron una puntuación mejor, la diferencia estadísticamente no es significativa (Tabla 1 ).

Concluimos entonces que, tanto el grupo control como el grupo experimental empiezan en las mismas condiciones en las tres dimensiones evaluadas, antes de la aplicación del programa de los juegos "DIMAPA".

Tabla 2.

Comparación de la Atención Selectiva post-test entre el Grupo Control y Experimental.

\begin{tabular}{llll}
\hline \multirow{2}{*}{$\begin{array}{l}\text { Atención Selectiva } \\
\text { Medición Post-test }\end{array}$} & \multicolumn{2}{c}{ Grupo de Estudio } \\
\cline { 2 - 4 } Efectividad Total & Media Aritmética & 479.00 & 533.23 \\
& Valor Mínimo & 380 & 382 \\
& Valor Máximo & 652 & 641 \\
\cline { 2 - 4 } Concentración & P & $0.034(\mathrm{P}<0.05)$ S.S. \\
\hline \multirow{5}{*}{ Variación } & Media Aritmética & 177.91 & 212.46 \\
& Valor Mínimo & 70 & 156 \\
& Valor Máximo & 293 & 283 \\
\cline { 2 - 4 } & P & $0.023(\mathrm{P}<0.05)$ S.S. \\
\hline & Media Aritmética & 16.00 & 13.62 \\
& Valor Mínimo & 1 & 2 \\
\hline & Valor Máximo & 26 & 28 \\
\cline { 2 - 4 } & P & $0.048(\mathrm{P}<0.05)$ S.S. \\
\hline
\end{tabular}

Matriz de datos recolectados. 
En la tabla 2 en lo que se refiere a comparación de la atención selectiva post-test entre el grupo control y experimental, con respecto a la efectividad total, los estudiantes del grupo control obtuvieron una puntuación de 479; mientras los estudiantes del grupo experimental obtuvieron una puntuación de 533.23; esta diferencia de puntuaciones es estadísticamente significativa.

Con respecto a la concentración, los estudiantes del grupo control obtuvieron un puntaje de $177.91 \mathrm{y}$ los del grupo experimental 212.46, esta diferencia de puntuaciones es estadísticamente significativa.

En lo referente a la variación, los estudiantes del grupo control obtuvieron un puntaje de 16 y los del grupo experimental 13.62, esta diferencia es estadísticamente significativa.

Concluimos entonces que, al final del estudio, el grupo experimental obtuvo disminución de la variación y mayores puntajes tanto en la efectividad total como en la concentración respecto al grupo control, siendo la diferencia estadísticamente significativa; es decir, el uso de los juegos "DIMAPA" permite incrementar la efectividad total y concentración y por ende desarrollar la atención selectiva.

Resultados que son similares a los encontrados por Del Toro, et al. (2016), concluyen que la implementación de diversas actividades lúdicas, lograron aumentar la atención selectiva de los estudiantes.

\section{Discusión}

En relación a la hipótesis; los resultados demostraron la eficacia de los juegos "DIMAPA" para desarrollar la atención selectiva en los estudiantes de odontología con problemas atencionales.

Las actividades lúdicas diseñadas para el aprendizaje de la asignatura de Histología y Embriología lograron desarrollar significativamente la atención selectiva aumentando su efectividad total al ser evaluados con la prueba del Test "d2": TOT $0.034(\mathrm{P}<0.05)$ S.S. y elevando su nivel de concentración: $\mathrm{CON}$ $0.023(\mathrm{P}<0.05)$ S.S. De la misma forma el índice de variación tuvo una variación sustancial: VAR0. $048(\mathrm{P}<0.05)$ S.S. En los resultados hallados en el grupo experimental, según la media aritmética se observa la diferencia entre el pre-test de 479.00 y el post-test de 533.23 que obtiene el nivel más alto.

Se afirma entonces que el juego mejora la atención selectiva en los estudiantes de odontología con los resultados obtenidos del test $\mathrm{d} 2$ al comparar el pretest y post-test, tal como se aprecian en las tablas 1 y 2; de la cual se interpreta que antes de aplicar los juegos "DIMAPA" en el pre-test no muestran diferencias significativas, sin embargo, en el posttest se evidencian diferencias significativas entre el grupo control y grupo experimental luego de la ejecución de actividades lúdicas.

Estas actividades desarrolladas fueron evaluadas con el test d2. Según Brickenkamp (2009). El test que midió estos resultados es el d2 y pertenece a la categoría de los instrumentos que pretenden medir los procesos básicos necesarios para tener éxito en las tareas complejas, procesos que han sido denominados con los términos como atención, concentración mental, esfuerzo o control atencional. Por lo tanto, este instrumento de evaluación es una medida concisa de la atención selectiva y la concentración mental; tal como se describe en el manual del test $\mathrm{d} 2$, que alude a una selección de estímulos enfocada de modo continuo a un resultado.

De los resultados obtenidos se infiere que los estudiantes del grupo experimental, durante la aplicación del programa de actividades con los juegos "DIMAPA" fueron desarrollando habilidades que conforman la competencia para atender, tales como: a) La velocidad o cantidad de trabajo, que viene representado por el número de estímulos que se han procesado en un tiempo determinado (aspecto motivacional o intensidad de atención). b) La calidad de trabajo, que viene dado por el grado de precisión (aspecto de control de la atención), y c) La relación entre la velocidad y la precisión, lo que permite obtener conclusiones sobre el comportamiento, grado de actividad, estabilidad y consistencia, fatiga y eficacia de la inhibición atencional.

La presente investigación, a partir de los resultados nos permite establecer comparaciones con otros estudios; como la investigación que realizó Lemus (2016) quien hizo un estudio de la efectividad de un programa sobre la atención en niños de 6to grado de primaria, en una institución de Guatemala. Para la muestra se tomó en cuenta 
20 estudiantes de ambos sexos que comprenden la edad de 11-12 años, de nivel socio-económico medio. Para medir la atención selectiva utilizó el test $\mathrm{d} 2$ de Brickencamp. De manera creativa quien diseñó luego aplicó el programa "atento", que tuvo el objetivo de mejorar la atención selectiva. En el análisis de los resultados hizo uso de la comparación de media con la prueba estadística de T de Student a través del programa Excel. Concluyendo que los estudiantes de la investigación, aumentaron significativamente el nivel de atención selectiva, debido a los efectos del programa "atento".

De la misma manera, existe otra investigación como la de Joao (2010), que aplicó el programa de intervención educativa para aumentar la atención y la reflexividad PIAAR-R, en 37 niños entre 10 y 12 años con déficit de atención, alta impulsividad e hiperactividad. No encontrando efectos significativos en la atención y reflexividad, indicó que estos resultados pudieron estar relacionados con las medidas de evaluación del programa, factores temporales (frecuencia y ritmo de las sesiones), factores inherentes al técnico (falta de estrategias de control de calidad de intervención), la aparición de variables parásitas, como la aparición de fenómenos de influencia social, aspectos que conciernen a observadores/evaluadores externos y aspectos relacionados con las bases teóricas del programa.

Finalmente, la mejora de la atención selectiva del grupo experimental, demostrada estadísticamente, 38 se debe a la eficacia de los juegos "DIMAPA" que desarrolló la atención selectiva en los estudiantes de odontología. Se puede considerar que esta investigación constituye un aporte para realizar nuevos estudios sobre la atención.

\section{Conclusiones}

Los resultados demostraron que, la aplicación de los juegos "DIMAPA" influyen favorable y significativamente en el desarrollo de la atención selectiva en los estudiantes del grupo experimental; estos juegos al mismo tiempo actúan como mediadores en los procesos de enseñanza aprendizaje, logrando en los estudiantes una mejor comprensión de conceptos teóricos, optimizando así, sus competencias en el ámbito académico.

\section{Agradecimiento}

Nuestro agradecimiento a los estudiantes del segundo semestre de la Escuela Profesional de Odontología de una Universidad Privada con sede en Arequipa.

\section{Referencias biográficas}

Alsuhaymi, D. y Alzebidi, A. (2019). Saudi Teachers' Perceptions Regarding Adopting Digital Games in Teaching Practice. Turkish Online Journal of Educational Technology TOJET, 18(4), 62-69. Recuperado de: http:// search.ebscohost.com/login.aspx?direct=true $\underline{\mathrm{db}=}=$ eric \&AN $=\mathrm{EJ} 1232268 \&$ lang $=\mathrm{es} \&$ site $=$ eds-live \&scope $=$ site

Arias, F. (2012). El proyecto de investigación, introducción a la metodología científica. 6ta ed. Venezuela: Editorial Episteme.

Baena-Paz, G. (2017). Metodología de la investigación serie integral por competencias. 3ra ed. Grupo Editorial Patria. Recuperado de http://ebookcentral.proquest.com Created from biblioteca jsp on 2018-07-30 15:51:39.

Barragán, R., Lewis, S., Palacio, J. (2007) Autopercepción de cambios en los déficits atencionales intermedios de estudiantes universitarios de Barranquilla sometidos al Método de Autocontrol de la Atención método Mindfulness. Revista Cientifica Salud Uninorte, 23(2), 184-192

Brickenkamp, R. (2009). Manual Test de atención d2. Adaptación española: Seisdedos Cubero, N. 3ra ed. TEA ediciones, S.A. Madrid: España.

Bunge, M. (1976) La Investigación Científica. Barcelona. Ediciones Ariel.

Calatayud, E., Plo, F. y Muro, C. (2020). Análisis del efecto de un programa de estimulación cognitiva en personas con envejecimiento normal en Atención Primaria: ensayo clínico aleatorizado. Atención Primaria, 52(1), 3846. Doi: 10.1016/j.aprim.2018.09.007.

Calvo-Hernando, P. y Gómez-Gómez, M. (2018). Aprendizaje y juego a lo largo de Historia. $L a$ razón histórica, Revista hispanoamericana de Historia de las Ideas, 40, 23-31. Recuperado de https://dialnet.unirioja.es/servlet/ articulo? codigo $=6906464$ 
Cedeño Meza, J. y Loor Domo, E. (2019). Procesos de atención $y$ funciones ejecutivas en estudiantes de educación superior. Espirales, Revista Multidisciplinaria de Investigación, 3(29), 33-47. Doi: 10.31876/er.v3i29.589.

Cherrier, S., Le Roux, P. Y., Gerard, F. M., Wattelez, G., y Galy, O. (2020). Impact of a neuroscience intervention (NeuroStratE) on the schoolperformance of high schoolstudents: Academic achievement, self-knowledge and autonomy through a metacognitive approach. Trends in Neuroscience and Education, 18. Doi: 10.1016/j.tine.2020.100125. Recuperado de http://search.ebscohost.com/login.aspx?di rect $=$ true \&db=edselp\&AN $=\mathrm{S} 221194932030$ 0016\&lang $=$ es\&site $=$ eds-live \&scope $=$ site

Cid-Sillero, S., Pascual-Sagastizabal, E. \& Martínez-de-Morentin, J. I. (2020). Influence of self-esteem and attention on the academic performance of ESO and FPB students. Revista de Psicodidáctica (English Ed.), 25(1), 59-67. Doi: 10.1016/j.psicoe.2019.10.001. Recuperado de http://search.ebscohost.com/ login.asp $\mathrm{x}$ ?direct $=$ true $\& \mathrm{db}=$ edselp $\& \mathrm{AN}=$ $\underline{\text { S2530380519300243\&lang }=\text { es\&site }=\text { eds- }}$ live\&scope $=$ site

De la Cruz, P., Bello, E., Enrique, L., Baquero, M., Eduardo, L., y Estrada, E. (2020). Juegos de mesa para la investigación participativa: una etnografía experimental sobre el comercio de productos de la chagra en comunidades indígenas de la Amazonía colombiana. Revista de Estudios Sociales, 72, 33-46. Doi: 10.7440/res 72.2020.03

Del Toro, C., Álvarez, T. y Medina, J. (2016). Estrategias lúdicas para mejorar la atención de los estudiantes del grado de $3^{\circ}$ de la sede principal de la institución educativa Marceliano Polo. (Tesis de especialidad). Fundación Universitaria los Libertadores, Colombia. Disponible en https:// repository.libertadores.edu.co/bitstream/ handle/11371/759/EspitiaMedinaJuliaEdith. pdf? sequence $=2 \&$ is Allowed $=\mathrm{y}$

Falconi, Alajo, Cueva, Mendoza, Ramírez \& Palma (2017). Las neurociencias. Una visión de su aplicación en la educación. Revista Órbita Pedagógica, 4(1) 61-74. Recuperado de http://revista.isced-hbo.ed.ao/rop/index. $\mathrm{php} / \mathrm{ROP} / \mathrm{article} / \mathrm{view} / 89 / 85$

Flores, E. (2016). Proceso de la Atención y su Implicación en el Proceso de Aprendizaje. RevistaDidasc@lia: DidácticayEducación, 7(3), 187-199. Recuperado de http://search. ebscohost.com/login.aspx ?direct $=$ true $\& \mathrm{db}$ $=$ fap \&AN $=139253461 \&$ lang $=$ es \&site $=$ eds$\underline{\text { live \&scope }=\text { site }}$

Gago-Galvagno L., y Elgier, A., (2018). Trazando puentes entre las neurociencias y la educación. Aportes, límites y caminos futuros en el campo educativo. Psicogente, 40, 476. Doi: 10.17081/psico.21.40.3087. Recuperado de http://search.ebscohost.com/login. aspx?direct $=$ true $\& \mathrm{db}=$ edsdoj\&AN=edsdoj. b02f1a86e5744d6b89efb576a511c10b\&lang $=$ es\&site $=$ eds-live \&scope $=$ site

Gall, S., Adams, L., Joubert, N., Ludyga, S., Müller, I., Nqweniso, S., Pühse, U., du Randt, R., Seelig, H., Smith, D., Steinmann, P., Utzinger, J., Walter, C. y Gerber, M. (2018). Effect of a 20-week physical activity intervention on selective attention and academic performance in children living in disadvantaged neighborhoods: A cluster randomized control trial. PLoS ONE, 13(11), pp.1-18. Doi: 10.1371/journal.pone.0206908

Gargallo, B. (2000). Programa de intervención educativa para aumentar la atención y la reflexibilidad. (2a ed.). Madrid: TEA Ediciones.

Girgin, M. (2017). Use of Games in Education: GeoGuessr in Geography Course. Online Submission, 1(1), 1-6. Recuperado de: http:// search.ebscohost.com/login.aspx?direct=true $\& \mathrm{db}=$ eric \&AN $=$ ED 581261\&lang $=$ es \&site $=\mathrm{e}$ ds-live \&scope $=$ site

Hernández, R., Fernández, C. y Baptista, M. (2014). Metodología de la Investigación. México: Mc Graw Hill.

Holgado, Morlans B. y Díaz Alonso, L. (2015). Evaluación de un programa psicopedagógico para la mejora de la atención en estudiantes de educación primaria. Revista Española de Orientación y Psicopedagogía, 26(3), pp.26-44. DOI: doi: 10.5944/reop.vol.26. num.3.2015.16399

Huizinga, J. (1972). Homo ludens. Bs. As.: Alianza Editorial.

Introzzi, I., Aydmune, Y., Zamora, E. V., Vernucci, S. y Ledesma, R. (2019). Mecanismos de desarrollo de la atención selectiva en población infantil. (Spanish). Revista CES Psicología, 12(3), 105-118. Recuperado en http://search.ebscohost.com/login.aspx?direc $\mathrm{t}=$ true \& $\mathrm{db}=\mathrm{edb} \& \mathrm{AN}=139483921$ \&lang $=\mathrm{es} \&$ site $=$ eds-live \&scope $=$ site

Jiménez, E., Broche, Y., Hernández, A. y Díaz, D. (2019). Funciones ejecutivas, cronotipo 
y rendimiento académico en estudiantes universitarios. Revista Cubana de Educación Superior, 38(2), 214-235. Recuperado de http://search.ebscohost.com/login.aspx?direc $\mathrm{t}=$ true $\& \mathrm{db}=$ fap \&AN $=137654391 \&$ lang $=$ es \& site $=$ eds-live \&scope $=$ site

João, C. (2010). Avaliação de um Programa de Intervenção Educativa para o Aumento da Atenção e da Reflexividade. (Tesis de Maestría), Instituto Superior de Ciências da Saúde da Norte, Portugal. Disponible en https://repositorio.cespu.pt/ handle/20.500.11816/147

Khan, Y. A., Ahamad, Z. y Kousar, S. (2013). Factors Influencing Academic Failure of University Students. International Journal of Educational Administration and Policy Studies, 5(5), 79-84. Recuperado de http:// search.ebscohost.com/login.aspx?direct=true $\underline{\mathrm{db}}=\mathrm{eric} \& \mathrm{AN}=\mathrm{EJ} 1084170 \&$ lang $=\mathrm{es} \&$ site $=$ eds-live \&scope $=$ site

Lemus, K. (2016). Efectividad de un programa de atención en niños de sexto primaria. (Tesis de grado). Universidad Rafael Landívar, Guatemala. Disponible en http://recursosbiblio.url.edu.gt/ tesiseortiz/2016/05/24/Lemus-Katherine.pdf

Loyola, R. (2016). Programa para mejorar la atención selectiva y concentración en niños de 11 y 12 años con problemas atencionales en una I. E. de Villa el Salvador, Lima, 2016. (Tesis de Maestría). Universidad César Vallejo, Perú. Disponible en http://repositorio. ucv.edu.pe/handle/UCV/8612

Marles, C., Peña s, P. y Gómez, C. (2018). La lúdica como estrategia para la educación y cultura ambiental en el contexto universitario. Revista UNIMAR, 2. Recuperado de: http:// search.ebscohost.com/login.aspx?direct $=$ tr ue \&db=edsdoj\&AN=edsdoj. $74285 \mathrm{f} 82214 \mathrm{f}$ d1a365418ca76c1006\&lang $=$ es \&site $=$ eds$\underline{\text { live\&scope }=\text { site }}$

Mas-Machuca, M., Jordan A. y Tresserres, C. (2019). Implementation of Game-based Learning in Higher Education: an example in HR Management. 5th International Conference on Higher Education Advances (HEAd'19), Editorial Universitat Politécnica de Valéncia. Doi: 10.4995/ HEAd19.2019.9369. Recuperado de: http:// ocs.editorial.upv.es/index.php/HEAD/ HEAD19/paper/viewFile/9369/4614
Mendieta, L., Gayrey, O., Valverde, M., y Vargas, J. (2019). Incidencia del juego de la rayuela en el desarrollo de la psicomotricidad. Espirales Revista Multidisciplinaria de Investigación, 3(30), 1-16. Doi: 10.31876/er.v3i30.612

Mocha-Bonilla, J., Coba, E., Barquin, C., y Castro, W. (2018). Effects of a recreational games program in the definition of laterality. Espacios, 39(23), 9p. Recuperado de: http://search.ebscohost.com/login.aspx? direct $=$ true $\& \mathrm{db}=$ edselc \& $\mathrm{AN}=$ edselc .2 52.0-85048225558\&lang $=$ es \&site $=$ edslive \&scope $=$ site

Montero, B. (2017). Experiencias Docentes. Aplicación de juegos didácticos como metodología de enseñanza: Una revisión de la literatura. Pensamiento Matemático, 1, 7592. Recuperado de https://dialnet.unirioja.es/ servlet/articulo? codigo $=6000065$

Morán, M., Ruvalcaba, S. y Ley Fuentes, M. (2014). Las buenas prácticas en la enseñanza de la Bacteriología Veterinaria en un Ambientes Virtual. Revista Iberoamericana de Producción Académica y Gestión Educativa, 1(1).

Olfers, K., y Band, G. (2018). Game-based training of flexibility and attention improves task-switch performance: near and far transfer of cognitive training in an EEG study. Psychological Research, 82(1), 186. doi:10.1007/s00426-017-0933-z

Otzen, T., Manterola, C., Rodríguez-Núñez, I. y García-Domínguez, Maricela. (2017). La Necesidad de Aplicar el Método Científico en Investigación Clínica: Problemas, Beneficios y Factibilidad del Desarrollo de Protocolos de Investigación. International Journal of Morphology, 35(3), 1031-1036. Doi: 10.4067/ S0717-95022017000300035.

Portellano, J. (2019). La atención ejecutiva. Cuadernos de Pedagogía, 498, 110-114.

Ramos, D, Bianchi, M, Rebello, E., y de O-Martins, M. (2019). Interventions with games in an educational context: Improving executive functions. Psicologia: Teoria e Prática, 21(2), 316-335. doi:10.5935/1980-6906/psicologia. v21n2p316-335

Rojas, J. y Rincón, C. (2015). Estudio descriptivo comparativo de las funciones ejecutivas frías $\mathrm{y}$ rendimiento académico en adolescentes. Revista Mexicana de Neurociencia, 16(1), 40-50. Recuperado de http://search. 
ebscohost.com/login.aspx ?direct $=$ true $\& d b$ $=$ asn \&AN $=118323739 \&$ lang $=$ es $\&$ site $=$ edslive\&scope $=$ site

Souza, R., Lorrayne, E., Duarte da Silva, Ramos de Souza, M., Borges, C. y Souza L. (2016). Atividades lúdicas como estratégia de educação em saúde com idosos. Revista Ciência Em Extensão, 3, 154-163. Recuperado de: http://search.ebscohost.com/login.aspx?di rect $=$ true $\& d b=$ edsdoj\&AN=edsdoj. $1882 \mathrm{cfbb}$ c2464671beb1c453ecb87dff\&lang $=$ es\&site $=$ eds-live\&scope $=$ site

Stevens, C. y Bavelier, D. (2012). The role of selective attention on academic foundations: A cognitive neuroscience perspective. Developmental Cognitive Neuroscience, 2(Supplement 1), S30-S48. Doi: 10.1016/j. den.2011.11.001

Véglia, A., y González, M. (2018). Intervención sobre las Funciones Ejecutivas (FE) desde el contexto educativo. Revista Iberoamericana de Educación (Versión Impresa), 78(1), 2742. Doi: 10.35362/rie7813269. Recuperado de http://search.ebscohost.com/login.aspx?di rect $=$ true \&db=fap\&AN=133276454\&lang $=\mathrm{e}$ s\&site $=$ eds-live \&scope $=$ site

Vergara, M. (2017). El juego: inicios e importancia en la educación. Unifé, 23, 5355. Doi: 10.33539/educacion.2017.n23.1169. Recuperado de: http://revistas.unife.edu.pe/ index.php/educacion/article/view/1169/1114

Vygotsky, L. (1966). El papel del juego en el desarrollo. Madrid: Aprendizaje Visor.

Zabala-Vargas, S. A., Ardila-Segovia, D. A., García-Mora, L. H. y de Benito-Crosetti, B. (2020). Aprendizaje basado en juegos (GBL) aplicado a la enseñanza de la matemática en educación superior. Una revisión sistemática de literatura. Formación Universitaria, 13(1), 13-26. Doi: 10.4067/ S0718-50062020000100013. Recuperado de http://search.ebscohost.com/login.asp $x$ ?direct $=$ true \&AuthType $=i p, s s o \& d b=$ fap \&AN $=141869206 \&$ lang=es \&site=ehost$\underline{\text { live } \& \text { scope }=\text { site } \& \text { custid }=\text { S } 9226804}$

Zaragoza, E., Orozco, L., Macías, J., Gutiérrez, R., de Alba, M., Villalobos, R., Cerda, R., Gutiérrez, A., Pérez, K., Núñez, M., Hernández, D., Navarro, C. \& Gómez, N. (2015). Estrategias didácticas en la enseñanza-aprendizaje: lúdica en el estudio de la nomenclatura química orgánica en alumnos de la Escuela Preparatoria Regional de Atotonilco. Educación Química, 27(1), 43-51. Doi: 10.1016/j.eq.2015.09.005. Recuperado de: http://search.ebscohost.com/ login.aspx ?direct $=$ true $\& \mathrm{db}=$ edselc $\& A N=$ eds elc.2-52.0-84955237908\&lang $=$ es\&site $=$ edslive\&scope $=$ site 